\title{
Optokinetic and Vestibular Stimulation Determines the Spatial Orientation of Negative Optokinetic Afternystagmus in the Rabbit
}

\author{
Vito E. Pettorossi, ${ }^{1}$ Pierangelo Errico, ${ }^{2}$ Aldo Ferraresi, ${ }^{2}$ and Neal H. Barmack ${ }^{3}$ \\ 1/nstitute of Human Physiology, University of Perugia, I-06100 Italy, 2Institute of Human Physiology of Catholic University, \\ Rome, I-06100 Italy, and ${ }^{3}$ Neurological Sciences Institute, Oregon Health Sciences University, Portland, Oregon 97201-3098
}

Prolonged binocular optokinetic stimulation (OKS) in the rabbit induces a high-velocity negative optokinetic afternystagmus (OKAN II) that persists for several hours. We have taken advantage of this uniform nystagmus to study how changes in static head orientation in the pitch plane might influence the orientation of the nystagmus. After horizontal OKS, the rotation axis of the OKAN II remained almost constant in space as it was kept aligned with the gravity vector when the head was pitched by as much as $80^{\circ}$ up and $35^{\circ}$ down. Moreover, during reorientation, slow-phase eye velocity decreased according to the head pitch angle.

Thereafter, we analyzed the space orientation of OKAN II after optokinetic stimulation during which the head and/or the OKS were pitched upward and downward. The rotation axis of OKAN II did not remain aligned with an earth vertical axis nor a head vertical axis, but it tended to be aligned with that of the OKS respace. The slow-phase eye velocity of OKAN II was also affected by the head pitch angle during OKS, because maximal OKAN II velocity occurred at the same head pitch angle as that during optokinetic stimulation.

We suggest that OKAN II is coded in gravity-centered rather than in head-centered coordinates, but that this coordinate system may be influenced by optokinetic and vestibular stimulation. Moreover, the velocity attenuation of OKAN II seems to depend on the mismatch between the space-centered nystagmus rotation axis orientation and that of the "remembered" head-centered optokinetic pathway activated by OKS.

Key words: optokinetic afternystagmus; eye movement; inertial coordinates; head position; optokinetic stimulus; slowphase eye velocity
Most of reflexive eye movements shows spatial constancy. In monkeys, optokinetic afternystagmus (OKAN) tends to align with the gravitational axis after off-vertical axis stimulation (Dai et al., 1991), and the rotation axis of the horizontal postrotatory nystagmus (PRN) remains fixed in space when the head is tilted (Merfeld et al., 1993; Angelaki and Hess, 1994). Also, in cats, the PRN axis tends to be fixed in gravity coordinates (Harris, 1987). It has been suggested that the velocity storage component of the eye reflexive movements is coded in a gravity-centered coordinate system (Angelaki et al., 1995; Wearne et al., 1997). However, in the rabbit, spatial constancy has been shown also in different reflexive eye responses: the cervico-ocular reflex (Pettorossi et al., 1987) and the vestibular-induced quick phases (Pettorossi et al., 1997). Conversely, in humans, the space reorientation of the PRN and OKAN is still a controversial issue (Gizzi et al., 1994; Fetter et al., 1996a,b).

To accomplish the transformation from head-centered to space-centered coordinates, information about head position in space is required. The otolith signal could be the most important input responsible for aligning optokinetic afternystagmus or postrotatory nystagmus with the direction of the gravity vector, because it provides information about static head position in space. However, the alignment of the reflexive eye responses may not be simply influenced by the otolithic signal, but other sensory information may be also important in modifying the response

\footnotetext{
Received May 19, 1998; revised Nov. 30, 1998; accepted Dec. 7, 1998.

This research was supported in part by the Consiglio Nazionale delle Ricerche, the Ministero dell'Universitá e della Ricerca Scientifica e Tecnologica, and the National Eye Institute.

Correspondence should be addressed to Vito Enrico Pettorossi, Istituto di Fisiologia Umana, Universitá di Perugia, Via del Giochetto, I-06100 Perugia, Italy. Copyright (C) 1999 Society for Neuroscience $\quad 0270-6474 / 99 / 191524-08 \$ 05.00 / 0$
}

orientation, inducing a change in the internal reference frame. In the previous experiments, the issue of a possible modification of the nystagmus orientation was not studied. The possible influence of nonvestibular sensory input in modifying response orientation can be addressed by using prolonged, binocular optokinetic stimulations. This would induce a plastic changes in the visuovestibular circuitry, which may affect the internal reference frame.

In the present paper, we investigated how optokinetic and gravitational stimulation influenced the nystagmus reference frame of rabbits. We used prolonged (24-48 hr) binocular optokinetic stimulation to establish a constant velocity nystagmus, termed negative optokinetic afternystagmus (OKAN II). The slow phase of OKAN II is opposite to the direction of the optokinetic stimulus and may reflect an adaptive process aimed at counteracting a persistent unidirectional retinal stimulation (Malcolm and Melvill-Jones, 1970; Brandt et al., 1974; Buttner et al., 1976; Waespe and Henn, 1978; Koenig and Dichgans, 1981; Leigh et al., 1981; Barmack and Nelson, 1987; Maioli, 1988). This sustained nystagmus would alleviate the interpretative difficulty of PRN and OKAN transient responses, because the trajectory of its slow phase could be monitored during changes of head orientation with respect to visual and gravitational coordinate systems.

After establishing OKAN II, we measured the rotation axis of its slow phase during different perturbations of the head about the interaural axis (pitch). Thereafter, we investigated the effect of different rotation angles of the optokinetic drum combined with different head pitch angles on the OKAN II orientation to ascertain whether the nystagmus would be always oriented in the horizontal plane of the earth or would change its orientation according to the plane of optokinetic stimulation. 


\section{MATERIALS AND METHODS}

Subjects. Thirteen pigmented rabbits were anesthetized with ketamine hydrochloride $(50 \mathrm{mg} / \mathrm{kg})$, xylazine $(6 \mathrm{mg} / \mathrm{Kg})$, and acepromazine maleate $(1.2 \mathrm{mg} / \mathrm{kg})$ to fix two stainless steel screws to the calvarium aligned in a stereotaxic apparatus so that the lambda suture was $1.5 \mathrm{~mm}$ above the bregma suture $\left(0^{\circ}\right.$ stereotaxic).

Optokinetic stimulation. Rabbits were placed in a restrainer at the center of a contour-rich patterned optokinetic drum (diameter of $110 \mathrm{~cm}$, height of $115 \mathrm{~cm}$ ). The direction of optokinetic stimulation (OKS) was in the posteroanterior direction [posterior $(\mathrm{P}) \rightarrow$ anterior $(\mathrm{A})$ ] with respect to the left eye. The head of the rabbit was fixed to the apparatus by a spring-loaded flexible coupling. This coupling attached to the implanted head screws. This device prevented lateral head displacement, permitting only small phasic sagittal movements, so that the imposed head position was maintained throughout the optokinetic stimulation. The body of the rabbit was encased in foam rubber and fixed with elastic straps to a plastic tube aligned with the longitudinal axis of the rate table. The method of restraint caused no pressure on any part of the body.

Rabbits received optokinetic stimulation for $48 \mathrm{hr}$ at $5^{\circ} / \mathrm{sec}$ of drum velocity to evoke OKAN II with a relative constant velocity (Barmack and Nelson, 1987). The rabbit was able to maintain its normal posture with all four paws in contact with the support surface. Electrocardiogram and respiratory rate were monitored and remained within the normal range. Any unexpected stimulus disturbing the rabbit induced an increase of respiratory and cardiac frequency. Every $8 \mathrm{hr}$, the rabbits were removed from the apparatus for $30 \mathrm{~min}$ and given food and water. The feeding regimen was sufficient as indicated by the maintenance of the body weight. Rabbits easily adapted to the restraint and showed no reluctance to resubmit to restraint after the brief feeding intervals.

Head and stimulus orientation during conditioning procedure. The spring-loaded flexible coupling that attached the head to the restrainer was positioned at an angle of $12^{\circ}$ in the posteroanterior direction. This angle aligned the horizontal semicircular canals so that they were orthogonal to the direction of gravity $\left(0^{\circ}\right.$ pitch angle) (Barmack, 1987). This angle approximately corresponds to the natural head angle maintained by rabbits (Soodak and Simpson, 1988). During optokinetic stimulation, the head-body of the rabbit was positioned at different pitch angles with respect to earth vertical. The plane of rotation of the optokinetic drum could also be independently aligned with respect to earth vertical. Four rabbits were conditioned with the head pitched $0^{\circ}$ and the drum rotating in the horizontal plane (head pitch of $0^{\circ}$, OKS of $0^{\circ}$ ). Three rabbits received optokinetic stimulation with the drum pitched either $20^{\circ}$ upward [counterclockwise $(\mathrm{CCW})$ ] or $20^{\circ}$ downward [clockwise $(\mathrm{CW})]$ and the head maintained at $0^{\circ}$ pitch angle (head pitch of $0^{\circ}$, OKS of $\pm 20^{\circ}$ ). Three rabbits were stimulated with the head pitched $20^{\circ}$ $\mathrm{CCW}$ or $20^{\circ} \mathrm{CW}$ and the drum at $0^{\circ}$ (head pitch of $\pm 20^{\circ}$, OKS of $0^{\circ}$ ). Three rabbits were stimulated with the head pitched up $35^{\circ}(\mathrm{CCW})$ and the drum also pitched up at $35^{\circ}$ (head pitch of $+35^{\circ}$, OKS of $+35^{\circ}$ ).

Eye movement recording. After $48 \mathrm{hr}$ of optokinetic stimulation, the rabbits were placed in the dark, and the head was attached to a rigid restraining bar pitched downward $12^{\circ}$ in the posteroanterior direction. Eye movements were measured by infrared light projection technique. The right eye was topically anesthetized with proparacaine hydrochloride, and a small suction cup (diameter of $3 \mathrm{~mm}$, weight of $135 \mathrm{mg}$ ) bearing a light-emitting diode (LED) was attached to the right eye so that the axis of light was aligned with the visual axis (Barmack and Nelson, 1987). The narrow beam of infrared light was detected by a photosensitive $x-y$ position detector (SC 50; United Detector Technology, Hawthorne, CA), which was fixed relative to the head. The circular photosensitive $x-y$ position detector had a diameter of 1.5 inches and gave a continuous $x-y$ voltage proportional to the position of the incident centroid of infrared light. The eye movement recording system was calibrated by moving the LED on a model of the rabbit eye through a known angular displacement. The system had a sensitivity of $0.2 \mathrm{~min}$ of arc and was linear to within $5 \%$ for eye deviations of $\pm 15^{\circ}$ and to $8 \%$ for deviations of $\pm 30^{\circ}$. Analog signals of head and eye position were stored on a magnetic tape recorder (Store D4; Racal Recorders, Hythe, UK) and displayed on a digital storage oscilloscope (TDS 420; Tektronix, Marlow, UK). The spatial orientation of the OKAN II was studied by stepping the table at different static angles $\left(0 \pm 180^{\circ}\right)$ about the interaural axis. Step velocity ranged from 0.2 to $60^{\circ} / \mathrm{sec}$ and step acceleration from 2 to $300-350^{\circ} / \mathrm{sec}^{2}$. In the lateral-eyed rabbit, head pitch induces eye torsion and rotation vector shift in $\mathrm{CCW}$ and $\mathrm{CW}$ direction, in response to downward $(\mathrm{CW})$ and upward $(\mathrm{CCW})$ head pitch, respectively. The time course of changes in the plane of nystagmus was examined using steps of high velocity. In all the figures in this paper, downward and upward head pitches are indicated as $\mathrm{CW}$ and $\mathrm{CCW}$, respectively.

The eye position was expressed as a rotation vector $E=\tan (\rho / 2) u$ [where $u$ is the unity vector, and $\rho$ is the angle of rotation about $u$, (Haustein, 1989)] in the coordinate system referred to as standard head-fixed reference system: the head vertical coincided with gravity when the stereotaxic head position was pitched $12^{\circ}$ nose down. The spatial orientation of OKAN II with respect to the head vertical was reconstructed from its vertical and horizontal slow-phase eye velocity (SPEV) components and plotted as a function of head pitch angle. The horizontal and vertical components of the eye position vector were differentiated using the Savitzky and Golay smoothing method (Savitzky and Golay, 1964) to obtain the coordinate velocity, and then the angular eye velocity vector $\Omega$ was calculated $\left[\Omega=2 /(d E / d t+E x d E / d t) /\left(1+|E|^{2}\right)\right]$. The derivative was taken by averaging the slopes of two adjacent position data points (200 points/sec) of the horizontal and vertical components.

Average SPEV of both horizontal and vertical components was computed from multiple intersaccadic samples taken at $5 \mathrm{msec}$ intervals. Averaged values were used, because under some stimulus conditions, slow phases were curvilinear rather than constant velocity. This was especially true after large changes in head pitch angles. Usually, 20-30 consecutive slow-phase velocities values were averaged when their variability was $<5 \%(\sim 15 \mathrm{sec}$ after a step change in head position was completed). Single slow-phase velocity values were used only for describing the time course of changes in slow-phase velocity immediately after a step change in head position.

A first-order exponential decay $\left(y=y_{0}+A_{\mathrm{e}}{ }^{-\left(\mathrm{x}-\mathrm{x}_{0}\right) / \mathrm{t}}\right)$ was fitted to the eye responses to determine the time constants of the changes in the nystagmus orientation and velocity.

Statistical evaluation. Statistical evaluations were performed by using a $t$ test, and a difference was considered statistically significant at $p<0.05$. The goodness fit was established by $\chi^{2}$ for sine function and by correlation coefficient $(R)$ for exponential decay, 1 - and $2^{\circ}$-order polynomials. The fit was obtained by minimizing the mean square error between the data and the curve (Levenberg-Marquardt algorithm).

\section{RESULTS}

Rotation axis of OKAN II remains fixed in space during changes in head pitch angle

Four rabbits received $48 \mathrm{hr}$ of horizontal optokinetic stimulation $\left(\mathrm{P} \rightarrow \mathrm{A}\right.$; left eye) at $5^{\circ} / \mathrm{sec}$ with the head positioned at $0^{\circ}$ pitch angle (head pitch of $0^{\circ}$, OKS of $0^{\circ}$ ). After the stimulation was stopped, the rabbit was placed in the dark, and eye movements were monitored as OKAN II developed. When the animals were pitched, the rotation axis of the nystagmus remained aligned with the earth vertical by a simultaneous reorientation of both the slow and fast phases in the head (Fig. 1). There was a corresponding increase in the vertical component of SPEV as the horizontal component decreased.

The reorientation had a gain (angle of OKAN II rotation axis rehead vertical-head pitch angle) close to 1 within a range of pitch angle of $\sim 35^{\circ} \mathrm{CW}$ and $80^{\circ} \mathrm{CCW}$ (Fig. 2). Beyond this range, the reorientation showed a progressive reduction. The nystagmus reorientation over the whole range of pitch angles was fitted by a sinusoid. This sinusoid showed a phase shift of $\sim 20^{\circ}$ in $\mathrm{CCW}$ direction of head pitch angle and a vertical shift of $\sim 22^{\circ}$ in $\mathrm{CW}$ direction of rotation axis angle (Fig. 2, Table 1). The reorientation gains were not affected by the absolute value of the velocity of the nystagmus. The gains were similar in all the animals, although there were large differences in nystagmus velocity, ranging from 15 to $50^{\circ} / \mathrm{sec}$. The gains were not changed when the head was pitched slowly $\left(0.2^{\circ} / \mathrm{sec}\right)$ or when it was pitched abruptly $\left(40^{\circ} / \mathrm{sec}\right)$. In other words, the gain of OKAN II reorientation appeared to be dependent on activation of the otolithic receptors but not the vertical semicircular canals.

Beyond $90^{\circ}$ of pitch, the reorientation values deviated from a sinusoidal function, primarily in downward direction, because the nystagmus orientation did not decrease as much as the sine 

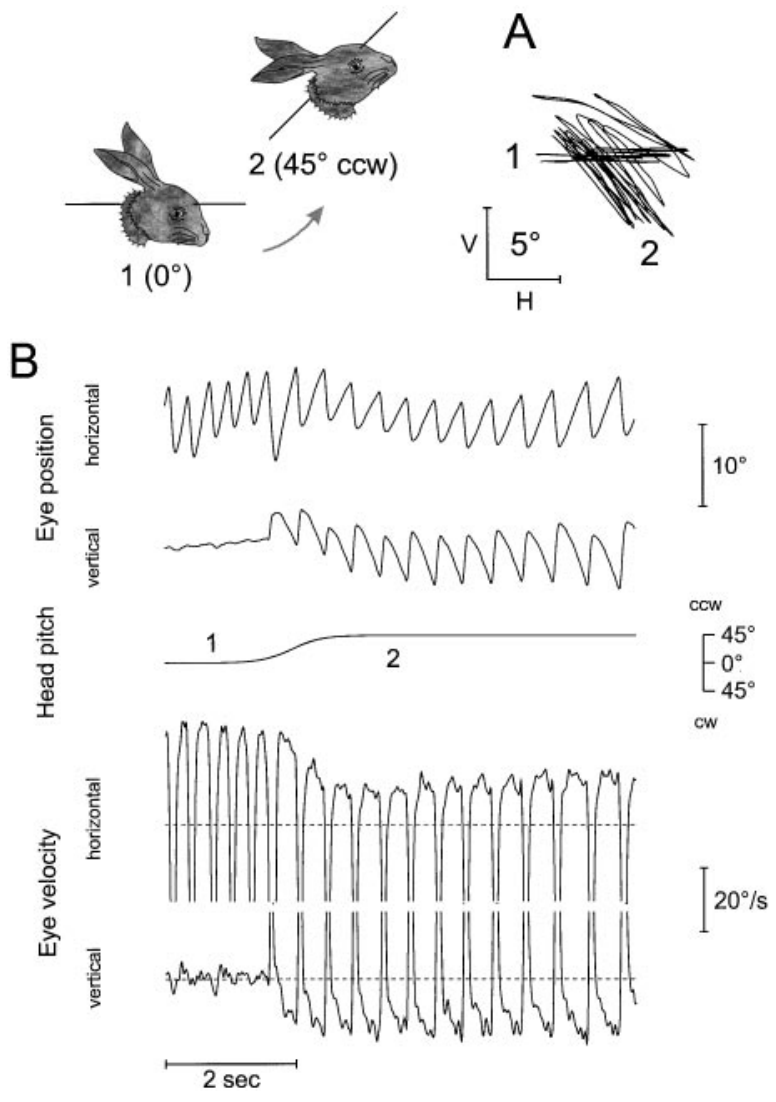

Figure 1. Shift in rotation axis of OKAN II induced by change in head pitch angle. $A$, Example of eye position record during OKAN II in which horizontal eye position is plotted against vertical eye position. Eye movements during OKAN II were restricted to a horizontal plane when the head pitch angle was maintained at $0^{\circ}\left(1\right.$, head pitch of $\left.0^{\circ}\right)$. When the head was pitched up $\left(2,45^{\circ} \mathrm{CCW}\right)$, the plane of eye movements shifted by approximately the same angle in $\mathrm{CW}$ direction to maintain the axis of eye movement vertical in space. $B$, Example of horizontal and vertical eye movements as a function of time. The top two traces represent horizontal and vertical eye position. The bottom two traces represent horizontal and vertical eye velocity. The middle trace represents head pitch angle.

function predicted (Fig. 2). The nystagmus had an abrupt inversion, because the vertical component changed direction within a few degrees of head pitch (Figs. 2, 3). This inversion occurred at $150 \pm 11^{\circ}$ in $\mathrm{CW}$ direction of head pitch. The rotation axis at the center of maximal nystagmus reorientation and that at the inversion point were displaced from the earth vertical by approximately the same amount $\left(20-30^{\circ}\right)$ in $\mathrm{CCW}$ direction.

Changes in the OKAN II rotation axis occurred over a time course of $<3 \mathrm{sec}$ after a step pitch displacement. The time constants of these changes varied between 0.5 and $1.2 \mathrm{sec}$ (Fig. 4).

In addition to the relative change in the vertical and horizontal velocity components, reorientation was accompanied by a reduction of the absolute SPEV. The time constant of this decay was longer than the reorientation time constant, having a value of $\sim 1.5 \mathrm{sec}$ in the CCW direction and $5.2 \mathrm{sec}$ in the $\mathrm{CW}$ direction. In $\mathrm{CW}$ head pitch, nystagmus decay in slow-phase velocity began when $70 \%$ of the reorientation was already completed. When the animals were pitched back to the $0^{\circ}$ position, the return of the slow-phase velocity to the value observed at $0^{\circ}$ pitch angle was much slower.

OKAN II slow-phase velocity had the maximal value at $0^{\circ}$ pitch angle and decreased progressively when the rabbits were pitched

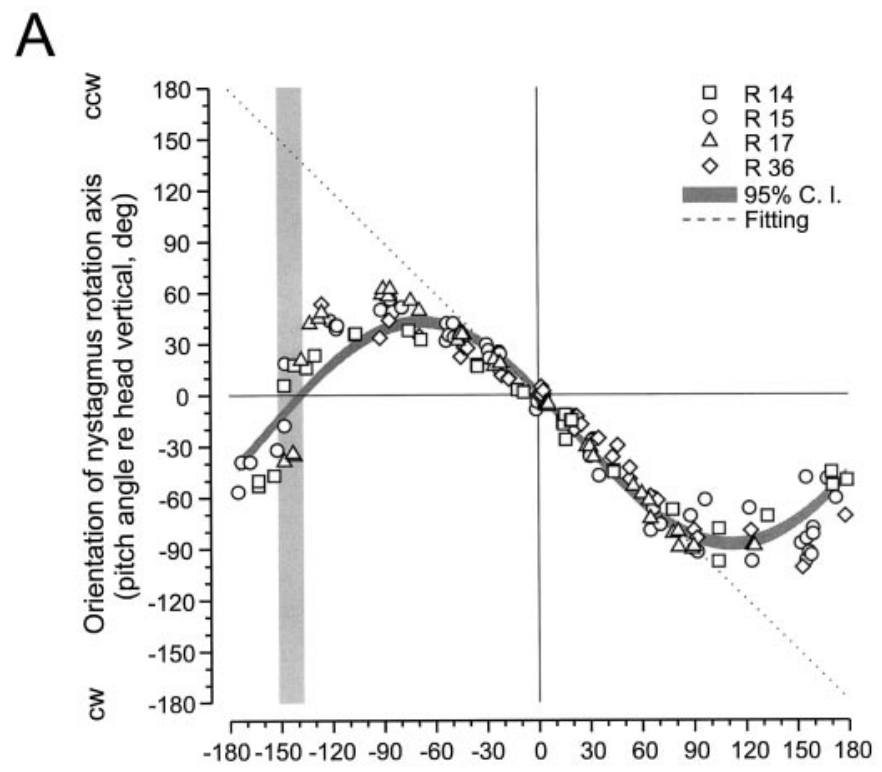

B

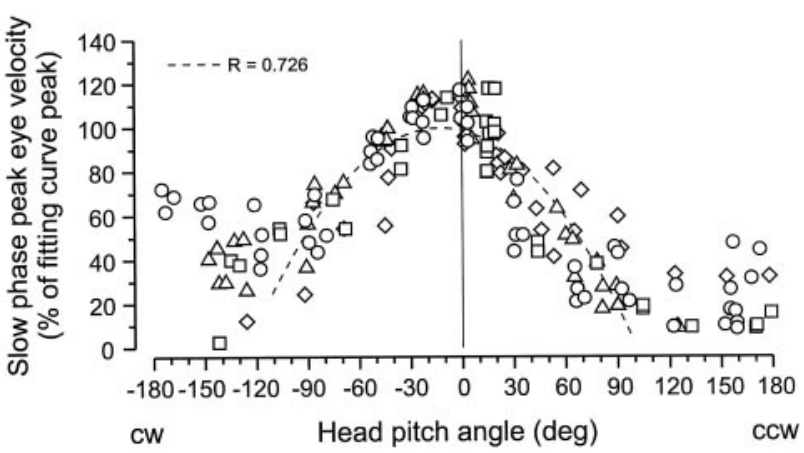

Figure 2. OKAN II rotation axis and velocity of slow phase are dependent on head pitch angle. $A$, In four rabbits, the rotation axis of OKAN II was measured while the head pitch angle was systematically varied. Each data point represents the mean of 20 consecutive slow-phase values. Data points were fitted with a sinusoid whose width comprises a $95 \%$ confidence interval $\left(\chi^{2}=123.5\right)$. The range over which an inversion of slow-phase eye movements occurs is indicated by the shaded vertical bar. $B$, Steady-state SPEV was measured at each head pitch angle (mean of 20 consecutive slow-phase values) and is fitted with a second-order polynomial within $\pm 90^{\circ}$ of head pitch angle.

upward and downward (Fig. 2). At $90^{\circ}$ (nose up), OKAN II slow-phase velocity was reduced by $90 \%$ of its maximal value at $0^{\circ}$. This decrease in OKAN II slow-phase velocity was greater in $\mathrm{CCW}$ head pitch than in $\mathrm{CW}$ pitch. A second-order polynomial fitting curve, evaluated within $\pm 90^{\circ}$, showed a peak value of $8 \pm$ $4^{\circ}$ shifted toward head pitch down (Fig. 2, Table 1).

\section{OKAN II induced by oblique optokinetic stimulation at $0^{\circ}$ head pitch angle}

Three rabbits received oblique OKS in upward direction $(\mathrm{P} \rightarrow \mathrm{A}$; down $\rightarrow$ up; left eye) at $5^{\circ} / \mathrm{sec}$, with the head maintained at $0^{\circ}$ pitch angle (head pitch of $0^{\circ}$, OKS of $+20^{\circ}$ ). Subsequently, the OKAN II, recorded from the right eye, was partially aligned with the OKS plane. When the head was at $0^{\circ}$ pitch angle, the rotation axis of the OKAN II was inclined $\sim 14^{\circ}$ in CCW direction (Fig. 5). In subsequent experiments, same rabbits received oblique OKS in downward direction $(\mathrm{P} \rightarrow \mathrm{A}$; up $\rightarrow$ down; left eye), with the head at $0^{\circ}$ pitch angle (head pitch of $0^{\circ}$, OKS of $-20^{\circ}$ ). The 
Table 1. Change in the rotation axis and slow-phase eye velocity of OKAN II

\begin{tabular}{|c|c|c|c|c|}
\hline \multirow[b]{2}{*}{ Head and stimulus pitch angle } & \multicolumn{3}{|c|}{ Sinusoidal curve fitting the plane of OKAN II } & \multirow{2}{*}{$\begin{array}{l}\begin{array}{l}\text { Second-order polynomial } \\
\text { curve fit of OKAN II velocity }\end{array} \\
\text { Phase of velocity peak (deg) }\end{array}$} \\
\hline & $\begin{array}{l}\text { Phase shift } \\
\text { (CCW, deg) }\end{array}$ & $\begin{array}{l}\text { Vertical shift } \\
(\mathrm{CW}, \mathrm{deg})\end{array}$ & Amplitude (deg) & \\
\hline $\mathrm{H} 0^{\circ}, \mathrm{OKS} 0^{\circ}(n=4)$ & $21.5 \pm 2.8$ & $22.1 \pm 2.6$ & $67.5 \pm 2.1$ & $8.0 \mathrm{CW} \pm 4.9$ \\
\hline $\mathrm{H} 0^{\circ}, \mathrm{OKS}+20^{\circ} \mathrm{CCW}(n=3)$ & $24.6 \pm 3.5$ & $5.2 \pm 1.7^{*}$ & $57.7 \pm 7.6$ & $3.7 \mathrm{CW} \pm 2.9$ \\
\hline $\mathrm{H} 0^{\circ}, \mathrm{OKS}-20^{\circ} \mathrm{CW}(n=3)$ & $25.3 \pm 4.1$ & $40.4 \pm 5.6^{*}$ & $65.3 \pm 3.7$ & $4.2 \mathrm{CW} \pm 4.1$ \\
\hline $\mathrm{H}+20^{\circ} \mathrm{CCW}, \mathrm{OKS} 0^{\circ}(n=3)$ & $19.3 \pm 2.3$ & $16.7 \pm 3.5$ & $69.7 \pm 1.5$ & $28.9 \mathrm{CCW} \pm 3.0^{*}$ \\
\hline $\mathrm{H}-20^{\circ} \mathrm{CW}, \mathrm{OKS} 0^{\circ}(n=3)$ & $18.6 \pm 3.7$ & $16.0 \pm 6.2$ & $67.0 \pm 2.1$ & $29.5 \mathrm{CW} \pm 4.1^{*}$ \\
\hline $\mathrm{H}$ and $\mathrm{OKS}+35^{\circ} \mathrm{CCW}(n=3)$ & $19.6 \pm 3.0$ & $2.3 \pm 2.3^{*}$ & $59.3 \pm 8.6$ & $30.7 \mathrm{CCW} \pm 5.2^{*}$ \\
\hline
\end{tabular}

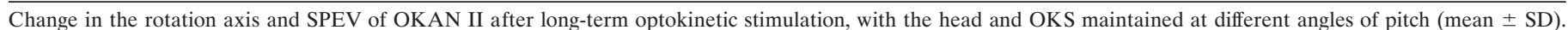

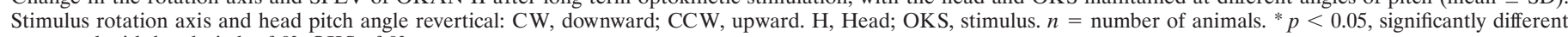
compared with head pitch of $0^{\circ}$, OKS of $0^{\circ}$.
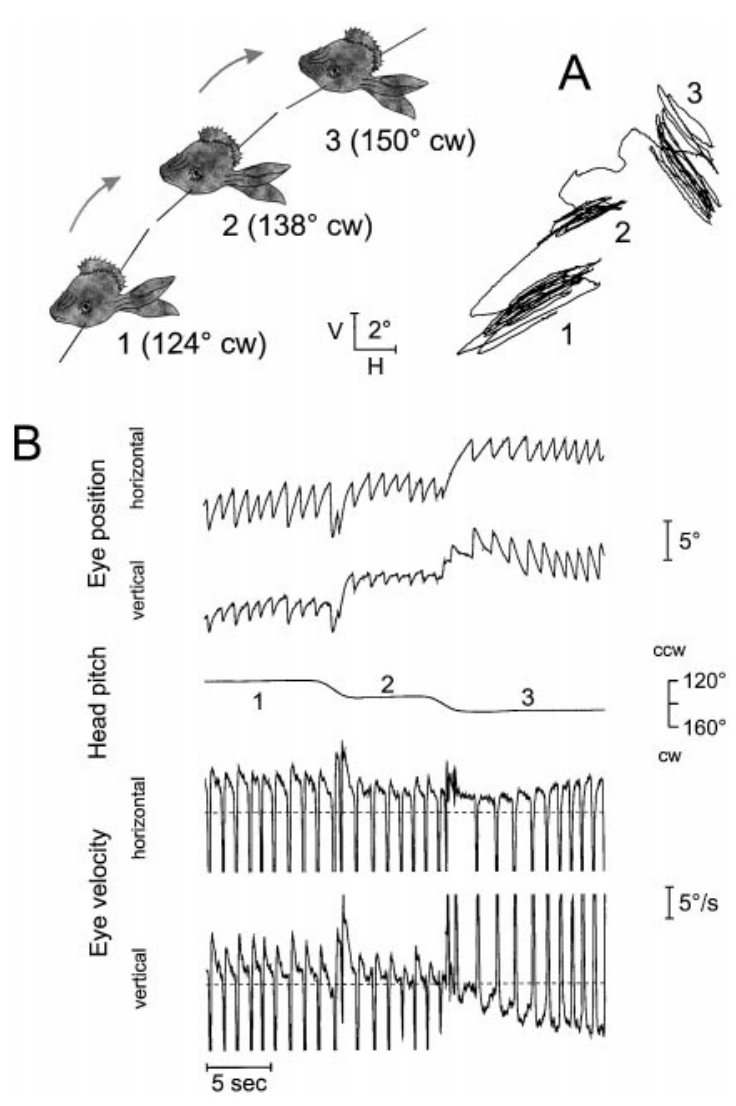

Figure 3. Abrupt shift in rotation axis of OKAN II induced by a small change in head pitch angle near supine orientation. When the head pitch angle changed by small increments near the angle of $135-150^{\circ}$ (CW direction), there was a large change in the axis of rotation of OKAN II of nearly $90^{\circ} . A$, The head pitch angle was changed from 124 (1) to 138 (2) and then to $150^{\circ}(3)$. Note that the axis of rotation of OKAN II changed abruptly when the head pitch angle changed from 138 to $150^{\circ}$. $B$, Examples of horizontal and vertical eye movements as a function of time. The top two traces represent horizontal and vertical eye position. The bottom two traces represent horizontal and vertical eye velocity. The middle trace represents head pitch angle.

rotation axis of the OKAN II was inclined $\sim 12^{\circ}$ in $\mathrm{CW}$ direction (Fig. 5). For both downward and upward stimulation, the sine curves fitting the orientation values over the whole range of head pitch angles were also shifted by almost the same amount in $\mathrm{CCW}$ and $\mathrm{CW}$ direction, respectively, as shown by their vertical shift (Fig. 5, Table 1), although there was no significant phase shift and no change in the inversion points. In addition, measurements of eye velocity indicated that the peak velocity for OKAN II evoked under both stimulus condition was almost the same (Fig. 5, Table 1).

\section{OKAN II induced by horizontal optokinetic stimulation at different head pitch angles}

Three animals received horizontal OKS with the head pitched $20^{\circ}$ in $\mathrm{CCW}$ and $\mathrm{CW}$ directions (head pitch of $\pm 20^{\circ}$, OKS of $0^{\circ}$ ). These stimulus combinations provided oblique OKS in retinal coordinates but horizontal OKS in space coordinates. Subsequently, OKAN II had rotation axis close to the earth vertical and tended to remain in earth vertical when the angle of head pitch was changed. In other words, OKAN II had the same characteristics as the OKAN II evoked by $0^{\circ} \mathrm{OKS}$ at $0^{\circ}$ head pitch angle (Fig. 6). No differences were observed for the inversion point. The slow phase of the OKAN II was not changed in either CCW or $\mathrm{CW}$ directions, as shown by the fitted sinusoid in Figure 6 and Table 1. However, the pitch angle of the head at which the maximal OKAN II slow-phase velocity occurred corresponded to the pitch angle of the head during optokinetic stimulation. If the head was pitched $\mathrm{CW}$ (head pitch of $-20^{\circ}$, OKS of $0^{\circ}$ ) during horizontal OKS, then the peak velocity occurred at $\sim 30^{\circ}$ in $\mathrm{CW}$ direction. When the animals were stimulated with the head pitched in upward position (head pitch of $+20^{\circ}$, OKS of $0^{\circ}$ ), the slow-phase velocity peak was at $\sim 29^{\circ}$ in $\mathrm{CCW}$ direction (Fig. 6, Table 1).

\section{OKAN II induced by combined inclination of optokinetic stimulation and head position}

Three rabbits received oblique optokinetic stimulation $\left(35^{\circ}\right.$; $\mathrm{P} \rightarrow \mathrm{A}$; down $\rightarrow$ up; left eye) while the head was pitched $35^{\circ}$ in $\mathrm{CCW}$ direction (head pitch of $+35^{\circ}$, OKS of $+35^{\circ}$ ). These stimulus combinations resulted in horizontal OKS in retinal coordinates but oblique OKS in space coordinates. Subsequently, the orientation of the OKAN II rotation axis in head coordinates was shifted $\sim 20^{\circ}$ in the $\mathrm{CCW}$ direction over the whole range of head pitch angles (Fig. 7) without significant phase shift. In addition, the peak velocity of the OKAN II was shifted $\sim 30^{\circ}$ in head pitch CCW direction (Fig. 7, Table 1).

\section{DISCUSSION}

\section{Spatial constancy of OKAN II}

We found that, in the rabbit, OKAN II showed spatial constancy, as do the cervico-ocular reflex (Pettorossi et al., 1987) and vestibular-induced quick phases (Pettorossi et al., 1997), sharing 


\section{A $0^{\circ} \rightarrow 45^{\circ}$ head pitch down (cw)}

Figure 4. Step change in head pitch angle induces a change in OKAN II rotation axis and a decrease in slow-phase velocity. Step changes in head pitch angle in both the $\mathrm{CW}(A)$ and $\mathrm{CCW}(B)$ directions from an initial horizontal head position (head pitch of $0^{\circ}$ ) caused both a decrease in absolute OKAN II slowphase velocity (dashed line) and a change in its horizontal (open squares) and vertical (open circles) velocity components. The $45^{\circ}$ step in either direction was executed within $1 \mathrm{sec}$, as indicated by the obliquely shaded bars in $A$ and $B$. The rotation axis of the nystagmus is indicated by the filled diamonds. Note that the change in the nystagmus rotation axis after a step change in the $\mathrm{CW}$ direction $(A)$ is substantially completed before there is a decrease in slow-phase velocity.
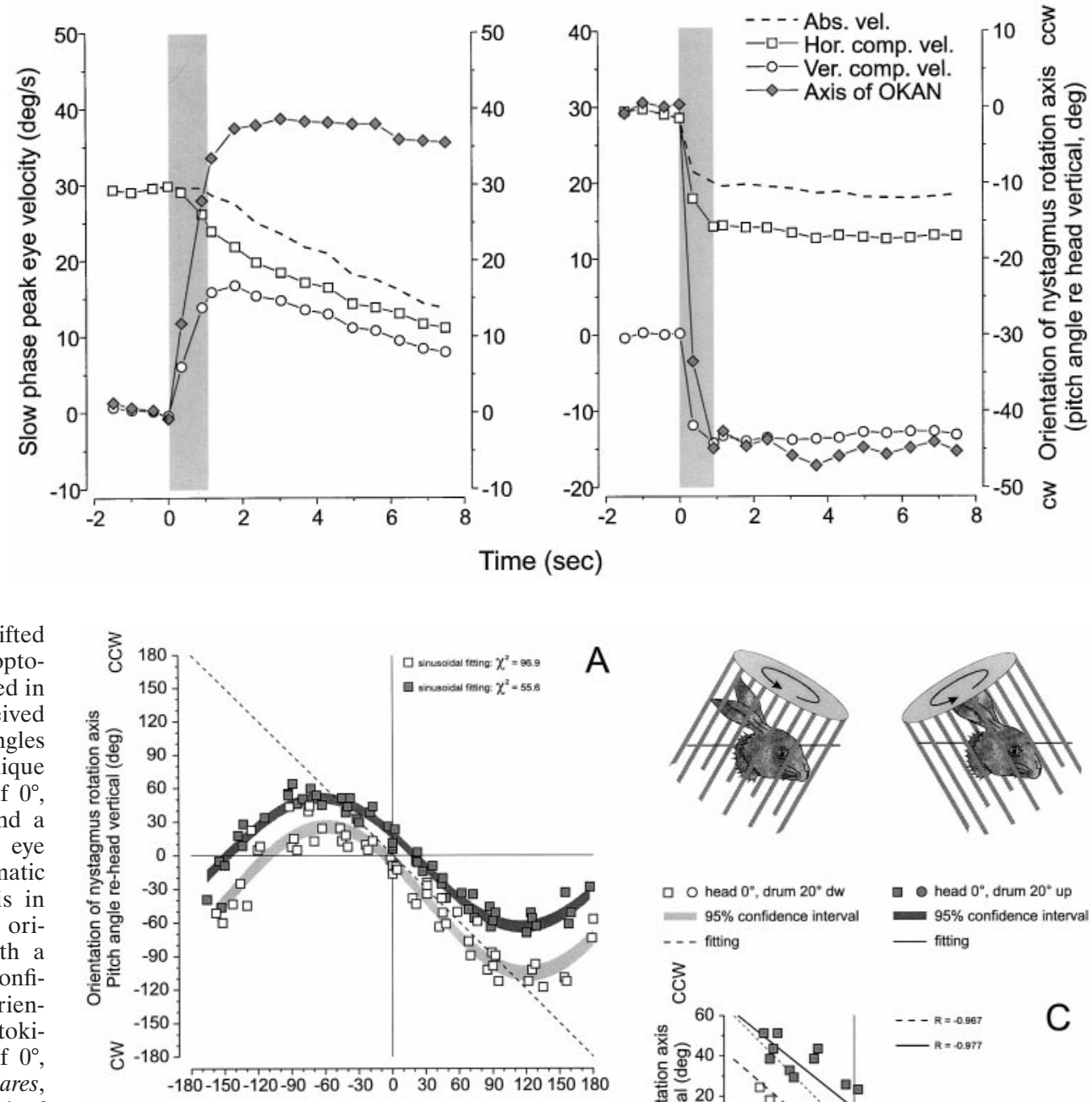

Figure 5. The OKAN II rotation axis is shifted by a change in the axis of rotation of the optokinetic stimulus when the head is maintained in a fixed horizontal orientation. A rabbit received optokinetic stimulation at two different angles with respect to the head: a downward oblique stimulation for the left eye (head pitch of $0^{\circ}$, OKS of $-20^{\circ}$; left schematic drawing) and a upward oblique stimulation for the left eye (head pitch of $0^{\circ}$, OKS of $+20^{\circ}$; right schematic drawing). $A$, The OKAN II rotation axis in head coordinates was plotted against head orientation, and data points were fitted with a sinusoid whose width comprises a $95 \%$ confidence interval. A shift of the nystagmus orientation is induced by the change in the optokinetic stimulation plane. For head pitch of $0^{\circ}$, OKS of $-20^{\circ}$, data points are open squares, fitted with a light gray sinusoid. For head pitch of $0^{\circ}$, OKS of $+20^{\circ}$, data points are filled squares, fitted with a dark gray sinusoid. $B$, Despite altering the zero crossing of the plane of OKAN II, the oblique optokinetic stimulation did not alter the head pitch angle (head pitch of $0^{\circ}$ ) at which the velocity of OKAN II was maximal. These velocities were fitted with a second-order polynomial for head pitch of $0^{\circ}$, OKS of $-20^{\circ}$ (open circles, dashed line) and head pitch of $0^{\circ}$, OKS of $+20^{\circ}$ ( filled circles, solid line). C, For clarity, the data illustrated in $A$ are plotted over a more
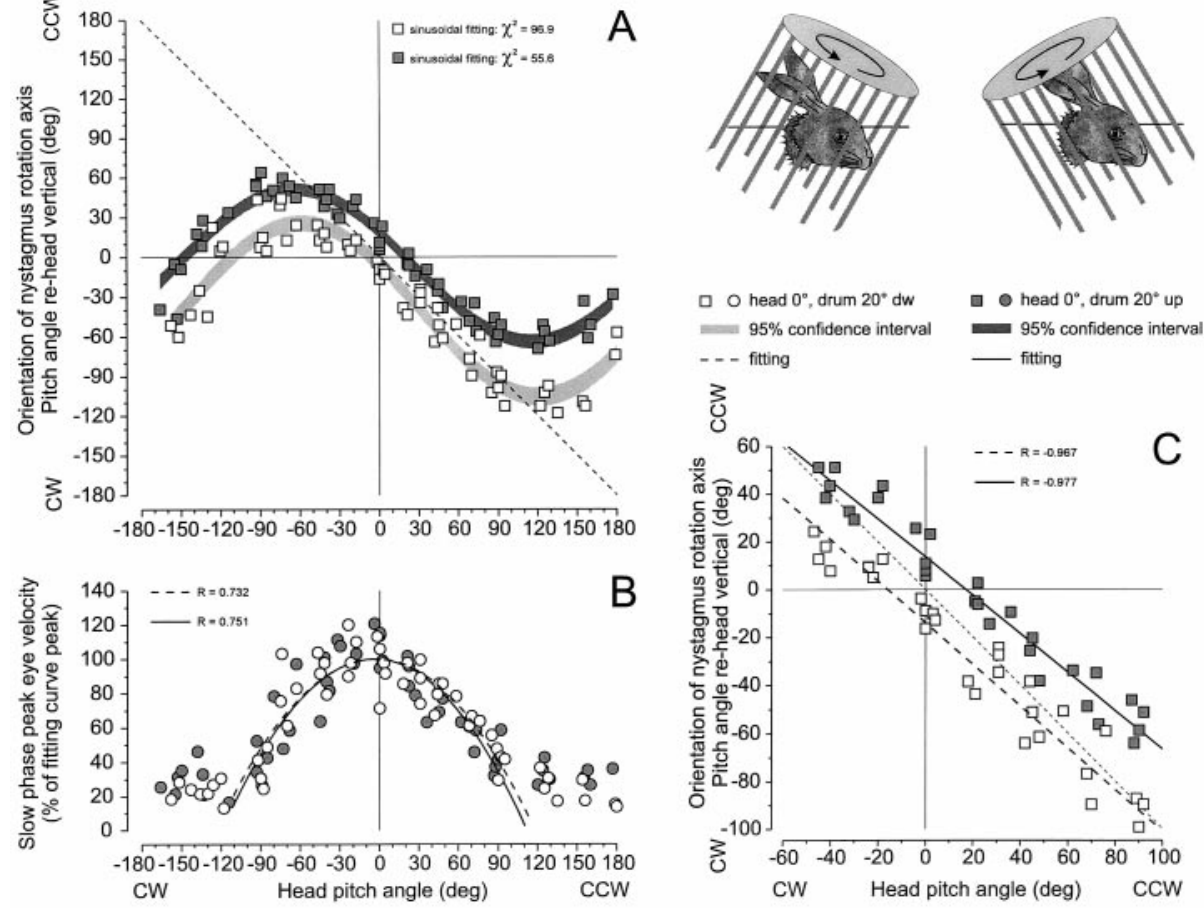

restricted range and fitted with linear regression lines. Data obtained after head pitch of $0^{\circ}$, OKS of $-20^{\circ}$ stimulation are represented as open squares and fitted with a dashed line. Data obtained after head pitch of $0^{\circ}, \mathrm{OKS}$ of $+20^{\circ}$ are represented as filled squares and fitted with a solid line.

with these reflexive eye movements a common space-centered reference system. The spatial constancy of OKAN II is not a peculiarity of the rabbit, because OKAN II reorientation has also been reported in the monkey, together with that of the OKAN I and PRN (Raphan and Cohen, 1988; Dai et al., 1991; Angelaki and Hess, 1994).

Although the gain of the OKAN II reorientation approaches that observed in the monkey during PRN (Angelaki and Hess, 1994), the time constant of the OKAN II reorientation is remarkably shorter. This may be attributable to the fact that OKAN II is a stable response, whereas PRN decays rapidly with a time constant of seconds. The decay of SPEV may increase the time needed to reorient the PRN in the orbit.
Another difference between eye movements of the monkey and rabbit may be related to the contribution of the tonic torsional reflex, which has an higher gain in the rabbit (Van der Steen and Collewijn, 1984) than in the monkey. Although we do not know how the pulling direction of the eye muscles would be affected by the eye torsion, the torsional reflex in the rabbit could make only a modest contribution to nystagmus reorientation. In fact, the maximal gain of torsional reflex is $\sim 0.3$, whereas the gain of OKAN II reorientation was 1.

The curve of the shift of the OKAN II plane within the orbit had sinusoidal shape over the whole range of pitch angles and was fitted by sine function. This may reflect the responses of the otolithic afferents to a full cycle pitch of the head, as previously 
Figure 6. SPEV of OKAN II is modified by a change in the head position maintained during the optokinetic stimulation. A rabbit received horizontal optokinetic stimulation at two different head pitch angles: head pitch of $-20^{\circ}$, OKS of $0^{\circ}$ (left schematic drawing) and head pitch of $+20^{\circ}$, OKS of $0^{\circ}$ (right schematic drawing). $A$, The OKAN II rotation axis in head coordinates was plotted against head orientation, and data points were fitted with a sinusoid whose width comprises a $95 \%$ confidence interval. For head pitch of $-20^{\circ}$, OKS of $0^{\circ}$, stimulation data points are open squares, fitted with a light gray sinusoid. For head pitch of $+20^{\circ}$, OKS of $0^{\circ}$, data points are filled squares, fitted with a dark gray sinusoid. Note the overlapping of the two sinusoids. $B$, Although there is no change in the OKAN II orientation, the different head positions altered the head pitch angle at which the velocity of OKAN II was maximal. These velocities were fitted with a second-order polynomial for head pitch of $-20^{\circ}$, OKS of $0^{\circ}$ (open circles, dashed line) and for head pitch of $+20^{\circ}$, OKS of $0^{\circ}$ ( filled circles, solid line). $C$, The data illustrated in $A$ are plotted over a more restricted range and fitted with linear regression lines. Data of head pitch of $-20^{\circ}$, OKS of $0^{\circ}$ stimulation are represented as open squares and fitted with a dashed line, and data of head pitch of $+20^{\circ}$, OKS of $0^{\circ}$ are represented as filled squares and fitted with a solid line.
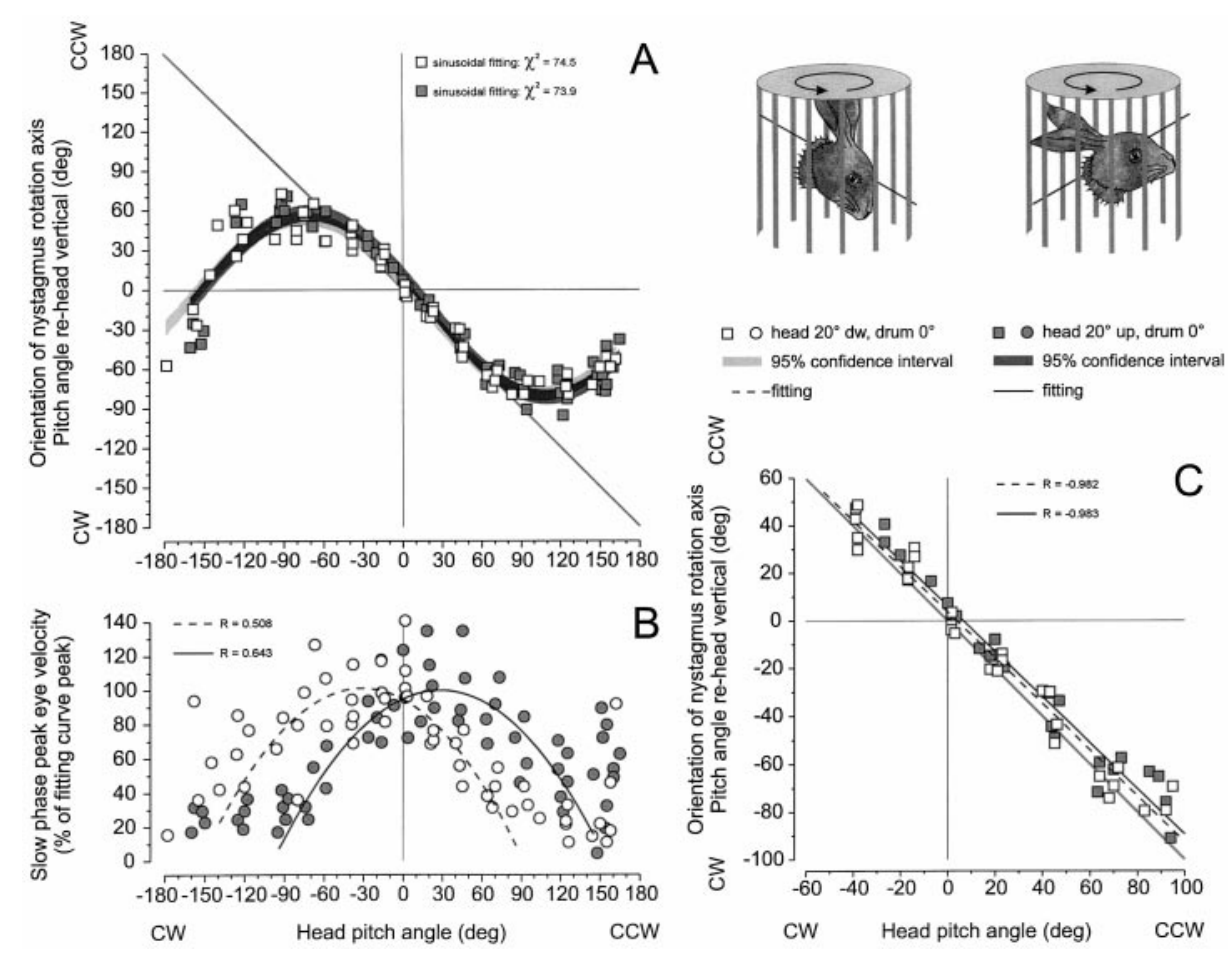

Figure 7. The rotation axis of OKAN II is shifted by a change in both the optokinetic rotation axis and the head position. A rabbit received oblique optokinetic stimulation (left eye) at head pitch angle with the same inclination: head pitch of $+35^{\circ}$, OKS of $+35^{\circ}$ (right drawing); and horizontal stimulation: head pitch of $0^{\circ}$, OKS of $0^{\circ}$ (left drawing). $A$, OKAN II rotation axis was plotted against head orientation, and data points were fitted with a sinusoid whose width comprises a $95 \%$ confidence interval. For the head pitch of $+35^{\circ}$, OKS of $0^{\circ}$, data points are filled squares, fitted with a dark gray sinusoid, and for head pitch of $0^{\circ}$, OKS of $0^{\circ}$, data points are open squares, fitted with a light gray sinusoid. After oblique head and stimulus inclination, the OKAN II rotation axis was shifted in CCW direction. $B$, The oblique head and stimulus inclination alters the head pitch angle (head pitch of $0^{\circ}$ ) at which the velocity of OKAN II was maximal. These velocities were fitted with a second-order polynomial for head pitch of $+35^{\circ}$, OKS of $+35^{\circ}$ ( filled circles, solid line) and head pitch of $0^{\circ}$, OKS of $0^{\circ}$ (open circles, dashed line). $C$, The data illustrated in $A$ are plotted over a more restricted range and fitted with linear regression lines. Data obtained after head pitch of $+35^{\circ}$, OKS of $+35^{\circ}$ stimulation are represented as filled squares and fitted with a solid line, and data after head pitch of $0^{\circ}$, $\mathrm{OKS}$ of $0^{\circ}$ stimulation as open squares and fitted with a dashed line. determined for both cat (Loe et al., 1973) and monkey (Fernandez et al., 1972). However, there may be disparities between otolithic responses and nystagmus reorientation. In fact, although the plane of the OKAN II, like the otolithic input, tended to vary as sine function of the head pitch angle, $>90^{\circ}$ of head pitch data deviated from sinusoidal fit. The angle of the plane of OKAN II did not progressively decrease as the head pitch angle approached $180^{\circ}$ (supine head position). This phenomenon is not present in the otolithic responses to pitch (Fernandez et al., 1972; Loe et al., 1973). It might represent a saturation of central neurons regulating reorientation of OKAN II that causes nystagmus to change its direction abruptly when the head is pitched slightly around the supine position. In addition, the center of the sinusoidal curve describing the reorientation, as well as the inversion point, lay on 
a plane $20-30^{\circ}$ out of that of horizontal semicircular canals, whereas the curve describing the averaged otolithic responses to pitch was centered at $0^{\circ}$ pitch (Loe et al., 1973). The lack of coincidence between the plane including the maximal modulation and inversion points with the plane of maximal otolithic receptor responsiveness may be a result of adaptation of the central reorienting process in response to functional needs. The reduced efficacy of downward reorientation could be related to the normal range of the pitch movements that, for the rabbit, is limited in downward direction by the ground.

\section{OKAN II orientation depends on the OKS orientation respace}

A second finding of this study concerns the space orientation of the OKAN II rotation axis. In contrast with previous studies showing the rotation axis of OKAN and PRN to be aligned with the gravity vector (Harris, 1987; Dai et al., 1991), we found that the plane of OKAN II is not invariably horizontal, but that it follows the plane of OKS respace, independently of the head position in space and the plane of OKS on the retina. This is clearly evident when both OKS and head are pitched during the optokinetic stimulation. In fact, the plane of OKAN II at $0^{\circ}$ head pitch angle is oblique. We believe that the difference between our results and those reported previously (Dai et al., 1991) is attributable to the duration of the optokinetic stimulation, which likely provokes plastic changes in the circuitry. In fact, OKAN II, which develops after $48 \mathrm{hr}$ of continuous stimulation, is not simply a reverberating response, like $\mathrm{PRN}$ or OKAN I; rather, it is a different event involving plastic changes in the optokinetic circuitry. To verify the influence of OKS duration, we also tried, in the rabbit, short-lasting optokinetic conditioning at various head and stimulus inclination (our unpublished observations), and we found that the afternystagmus was always horizontal. Therefore, in the case of prolonged optokinetic stimulation, plastic changes may be induced in the visuovestibular circuitry, not only for producing OKAN II but also for consolidating a new reference frame.

It has been proposed that nystagmus reorientation reflects characteristics of an internal reference frame for spatial orientation (Dai et al., 1991; Angelaki and Hess, 1994). In this view, we interpret the change in orientation of the OKAN II rotation axis away from an earth vertical alignment as a result of a change in the internal reference frame according to the orientation of the optokinetic stimulus in space. This might occur because the CNS interprets the orientation of prolonged optokinetic stimulation, which almost invariably occurs in the horizontal plane, as the real horizon.

\section{Changes in OKAN II slow-phase velocity induced by head pitch}

The absolute value of the OKAN II SPEV was also modified systematically by the head pitch, with a longer time constant than that of the nystagmus reorientation. The velocity was usually reduced when the head was tilted and came back when the head was tilted back, but there was a large difference in the velocity rising and falling timing. Decrease in nystagmus velocity may be attributable to an active inhibitory process, whereas the velocity increase may be a result of the inhibition removal and of the slower nystagmus velocity building up.

The change in slow-phase velocity at different head pitch angles resembles the change in velocity of PRN and OKAN I observed previously by others (Waespe et al., 1985). They showed that the time constants of OKAN I and PRN were shortened after head tilt away from the stimulation plane. The reduction of the absolute value of nystagmus velocity may minimize the sensory conflict between signals from optokinetic and semicircular canals and signals from otoliths (Guedry, 1965). More recently, it has been suggested that nystagmus slow-phase velocity reduction is necessary for the spatial transformation of both PRN and OKAN I (Angelaki and Hess, 1994). Our data conflict with this interpretation. In fact, in the present experiment, the time constant of the reorientation is actually shorter than that of the change in slowphase velocity, and the reorientation is maintained after nodulectomy without a parallel velocity reduction (Errico et al., 1996). Therefore, attenuation of nystagmus velocity is not necessary per se during a change in the plane of nystagmus but is an important accompanying phenomenon.

We suggest that the head pitch-induced change in velocity is related to the mismatch between the plane of activated retinal pathway coded in head-centered coordinates and the plane of OKAN II coded in a space-centered frame. In fact, when the OKS is horizontal and the head is pitched up or down, the peak velocity is shifted similarly in upward and downward direction so that maximal velocity occurs when the space-centered and headcentered planes coincided. The velocity dependence on the mismatch between the two planes implies also that the head-centered plane is stored in the CNS. Prolonged optokinetic stimulation induces long-lasting plastic changes along the optokinetic pathway to keep memory of optokinetic stimulus velocity. These "storage sites" include the retina (Liu et al., 1998), as well as the dorsal cap of the inferior olive (Barmack and Errico, 1993).

It is likely that the structures involved in the comparison between space-centered and head-centered responses include the nodulus and the ventral uvula. In studies on primates, this part of caudal vermis appears to be necessary for both spatial constancy and head tilt-induced velocity attenuation of the nystagmus (Waespe et al., 1985; Angelaki and Hess, 1995; Wearne et al., 1998). In the rabbit, the only effect of uvula-nodulus lesion was the complete disruption of the velocity modulation (Errico et al., 1996). This suggests that nodulus and uvula may be involved in detecting the differences between the space-centered plane in which the nystagmus occurs and the "remembered" plane of the activated optokinetic pathway coded in head coordinates.

In conclusion, our results indicate that the plane of OKAN II is coded in space-centered coordinates, and it is influenced by the plane of the OKS respace. The SPEV of OKAN II is also affected by the OKS orientation, and it is maximal when the plane of OKAN II coincides with the plane of the activated retinal pathway, coded in head-centered coordinates.

\section{REFERENCES}

Angelaki DE, Hess BJM (1994) Inertial representation of angular motion in the vestibular system of Rhesus monkeys. I. Vestibulo-ocular reflex. J Neurophysiol 71:1222-1249.

Angelaki DE, Hess BJM (1995) Inertial representation of angular motion in the vestibular system of Rhesus monkeys. II. Otolith-controlled transformation that depends on intact cerebellar Nodulus. J Neurophysiol 73:1729-1751.

Angelaki DE, Hess BJM, Suzuki J (1995) Differential processing of semicircular canal signals in the vestibulo-ocular reflex. J Neurosci 15: 7201-7216.

Barmack NH (1987) The influence of gravity on horizontal and vertical vestibulo-ocular and optokinetic reflexes in the rabbit. Brain Res 424:89-98.

Barmack NH, Errico P (1993) Optokinetically evoked expression of corticotropin-releasing factor in inferior olivary neurons of rabbits. J Neurosci 13:4647-4659. 
Barmack NH, Nelson BJ (1987) Influence of long-term optokinetic stimulation on eye movements of the rabbit. Brain Res 437:111-120.

Brandt T, Dichgans J, Buhele W (1974) Motion habituation: inverted self-motion perception and optokinetic afternystagmus. Exp Brain Res 21:337-352.

Buttner UW, Waespe W, Henn V (1976) Duration and direction of optokinetic after-nystagmus as a function of stimulus exposure time in the monkey. Arch Psychiatr Nervenkr 222:281-291.

Dai MJ, Raphan T, Cohen B (1991) Spatial orientation of the vestibular system. Dependence of optokinetic after-nystagmus on gravity. J Neurophysiol 66:1422-1439.

Errico P, Ferraresi A, Barmack NH, Pettorossi VE (1996) Role of cerebellar uvula-nodulus in the control of head orientation-specific eye velocity in the rabbit. Ann NY Acad Sci 781:614-618.

Fernandez C, Goldberg JM, Abend W (1972) Response to static tilts of peripheral neurons innervating otolith organs of the squirrel monkey. J Neurophysiol 35:978-987.

Fetter M, Heimberger J, Black R, Hermann W, Sievering F, Dichgans J (1996a) Otolith-semicircular canal interaction during postrotatory nystagmus in humans. Exp Brain Res 108:463-472.

Fetter M, Pfaff G, Heimberger J, Haslwanter T (1996b) 3D optokinetic responses in humans are not influenced by the gravito-inertial force vector. Soc Neurosci Abstr 434:1095.

Gizzi M, Raphan T, Rudolph S, Cohen B (1994) Orientation of human optokinetic nystagmus to gravity: a model-based approach. Exp Brain Res 99:347-360.

Guedry FE (1965) Orientation of the rotation axis relative to gravity: its influence on nystagmus and sense of rotation. Acta Otolaryngol (Stockh) 60:30-48.

Harris LR (1987) Vestibular and optokinetic eye movements evoked in the cat by rotation about a tilted axis. Exp Brain Res 66:522-532.

Haustein W (1989) Considerations on Listing's law and primary position by means of matrix description of eye position control. Biol Cybern 60:411-420.

Koenig E, Dichgans J (1981) Aftereffects of vestibular and optokinetic stimulation and their interaction. Ann NY Acad Sci 374:434-445.

Leigh RJ, Robinson DA, Zee DS (1981) A hypothetical explanation for periodic alternating nystagmus: instability in the optokinetic vestibular system. Ann NY Acad Sci 374:619-635.
Liu H, Qian ZY, Barmack NH (1998) Endogenous diazepam ligand in rabbit retina induced by long-term optokinetic stimulation and detected by differential display. Soc Neurosci Abstr 24:1024.

Loe PR, Tomko DL, Werner G (1973) The neural signal of angular head position in primary afferent vestibular nerve axons. J Physiol (Lond) 230:29-50.

Maioli C (1988) Optokinetic nystagmus: modeling the velocity storage mechanism. J Neurosci 8: 821-832.

Malcolm R, Melvill-Jones G (1970) A quantitative study of vestibular adaptation in humans. Acta Otolaryngol (Stockh) 70:126-135.

Merfeld DM, Young LR, Paige GD, Tomko DL (1993) Three dimensional eye movements of squirrel monkeys following postrotatory tilt. J Vestib Res 3:123-139.

Pettorossi VE, Errico P, Ferraresi A, Fedeli R (1987) Vestibular contribution to the orientation of cervico-ocular reflex in rabbit. Brain Res 403:58-65.

Pettorossi VE, Errico P, Ferraresi A (1997) Difference in quick phases induced by horizontal and vertical vestibular stimulations: role of the otolithic input. J Vestib Res 7:89-99.

Raphan T, Cohen B (1988) Organizational principles of velocity storage in three dimensions. The effect of gravity on cross-coupling of optokinetic after-nystagmus. Ann NY Acad Sci 545:74-92.

Savitzky A, Golay MJE (1964) Smoothing and differentiation of data by simplified least square procedure. Anal Chem 36:1627-1639.

Soodak RE, Simpson JI (1988) The accessory optic system of rabbit. I. Basic visual response properties. J Neurophysiol 60:2037-2054.

Van der Steen J, Collewijn H (1984) Ocular stability in the horizontal, frontal and sagittal planes in the rabbit. Exp Brain Res 56:263-274.

Waespe W, Henn V (1978) Reciprocal changes in primary and secondary optokinetic after-nystagmus produced by repetitive optokinetic stimulation in the monkey. Arch Psychiatr Nervenkr 225:23-30.

Waespe W, Cohen B, Raphan T (1985) Dynamic modification of the vestibulo-ocular reflex by the nodulus and uvula. Science 228:199-202.

Wearne S, Raphan T, Cohen B (1997) Contribution of vestibular commissural pathways to spatial orientation of the angular vestibuloocular reflex. J Neurophysiol 78:1193-1197.

Wearne S, Raphan T, Cohen B (1998) Control of spatial orientation of the angular vestibuloocular reflex by the nodulus and uvula. J Neurophysiol 79:2690-2715. 